\title{
Donor age and C1orf132/MIR29B2C determine age-related methylation signature of blood after allogeneic hematopoietic stem cell transplantation
}

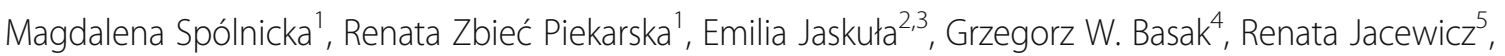 \\ Agnieszka Pięta ${ }^{1}$, Żanetta Makowska', Maciej Jedrzejczyk ${ }^{5}$, Agnieszka Wierzbowska ${ }^{6}$, Agnieszka Pluta ${ }^{6}$, \\ Tadeusz Robak ${ }^{6}$, Jarosław Berent ${ }^{5}$, Wojciech Branicki ${ }^{7}$, Wiesław Jędrzejczak ${ }^{4}$, Andrzej Lange ${ }^{3}$ and Rafał Płoski ${ }^{8^{*}}$
}

\begin{abstract}
Background: Our recent study demonstrated that DNA methylation status in a set of CpGs located in ELOVL2, C1orf132, TRIM59, KLF14, and FHL2 can accurately predict calendar age in blood. In the present work, we used these markers to evaluate the effect of allogeneic hematopoietic stem cell transplantation (HSCT) on the age-related methylation signature of human blood.

Methods: DNA methylation in $32 \mathrm{CpGs}$ was investigated in 16 donor-recipient pairs using pyrosequencing. DNA was isolated from the whole blood collected from recipients 27-360 days (mean 126) after HSCT and from the donors shortly before the HSCT.

Results: It was found that in the recipients, the predicted age did not correlate with their calendar age but was correlated with the calendar age $\left(r=0.94, p=4 \times 10^{-8}\right)$ and predicted age $\left(r=0.97, p=5 \times 10^{-10}\right)$ of a respective donor. Despite this strong correlation, the predicted age of a recipient was consistently lower than the predicted age of a donor by 3.7 years $\left(p=7.8 \times 10^{-4}\right)$. This shift was caused by hypermethylation of the C1orf132 CpGs, for C1orf132 CpG_1. Intriguingly, the recipient-donor methylation difference correlated with calendar age of the donor $\left(r=0.76, p=6 \times 10^{-4}\right)$. This finding could not trivially be explained by shifts of the major cellular factions of blood.

Conclusions: We confirm the single previous report that after HSCT, the age of the donor is the major determinant of age-specific methylation signature in recipient's blood. A novel finding is the unique methylation dynamics of Clorf132 which encodes MIR29B2C implicated in the self-renewing of hematopoietic stem cells. This observation suggests that C1orf132 could influence graft function after HSCT.
\end{abstract}

Keywords: Allogeneic hematopoietic stem cell transplantation, DNA methylation, Aging, Rejuvenation, MIR29B2C

Abbreviations: BM, bone marrow; HSCT, hematopoietic stem cell transplantation; IncRNA, long no-coding RNA

\section{Introduction}

It has been shown in animal models that elderly individuals exposed to a young systemic environment, for example, by surgical connection of the circulatory systems of young and old animal (heterochronic parabiosis), show reduced signs of biological aging in the cardiovascular,

\footnotetext{
*Correspondence: rploski@wp.pl

${ }^{8}$ Department of Medical Genetics, Warsaw Medical University, Pawińskiego

3c, Warsaw PL 02-106, Poland

Full list of author information is available at the end of the article
}

skeletal, and gastrointestinal as well as central nervous system [1-3]. Whereas parabiosis is a strictly experimental system, it has certain similarities with clinical state during an allogeneic transplantation including bone marrow transplant or the more often performed peripheral blood hematopoietic stem cell transplantation (HSCT).

Recently, we described a set of five CpG sites whose methylation in the whole blood predicts calendar age with high accuracy [4]. This set of predictors included 
CpG sites in ELOVL2 (6p24.2), C1orf132 (1q32.2), TRIM59 (3q25.33), KLF14 (7q32.3), and FHL2 (2q12.2). We developed a convenient method based on pyrosequencing for robust DNA methylation analysis in 32 CpGs located in these loci including the five best $\mathrm{CpG}$ sites included in the prediction model. Our approach allowed to predict actual age with mean absolute deviation (MAD) of 3.9 years and number of correct predictions $(+/-5$ years) that ranged depending on age category, from 87 to $50 \%$ [4].

The aim of this study was to evaluate the effect of HSCT on the age-related methylation signature of human blood obtained with set of $5 \mathrm{CpG}$ markers of age included in our age prediction model. In particular, we examined the effects of donor and recipient age as well as assessed whether differences existed in post-transplant methylation dynamics among individual CpG markers.

\section{Methods}

\section{Materials}

We studied peripheral blood samples from 16 pairs of donors and recipients. Transplant indications were in line with the European Group for Blood and Marrow Transplantation (EBMT) guidelines and the searching process was conducted according to the World Marrow Donor Association (WMDA) recommendations. Eleven patients suffered from acute myeloblastic leukemia, two from myeloproliferative diseases, one from $\mathrm{T}$-cell acute lymphoblastic leukemia, one from chronic myeloblastic leukemia, and one from paroxysmal nocturnal hemoglobinuria. Fourteen pairs were of the same sex; two males received a transplant from female donors. Samples from the donors included original samples collected for the purpose of HLA typing within 6 months before alloHSCT. Samples from the recipients reconstituted with hematopoietic stem cells of donor origin were collected at the median time of 91 days (range, 27-360) after alloHSCT. The general characteristics of recipients and donors are shown in Table 1. Briefly, the median age of recipients at the time of alloHSCT was 45 (range, 18-59, a detailed list of donors' and matched recipients' age is given in Additional file 1: Table S1). Twelve patients received myeloablative and four reduced-intensity conditioning. All patients underwent transplantation from unrelated donors. All patients but one were matched with recipients in 10/10 HLA alleles. All patients received peripheral blood stem cells as a transplant material. The median age of stem cell donors was 28 years (range, 20-63). The graft-versus-host disease prophylaxis included standard doses of cyclosporine (all patients) and methotrexate (12 patients) or mycophenolate mofetil (4 patients). Engraftment was achieved by day +20 in all the patients. In all recipients, full chimerism at the time of blood sample collection for methylation status analysis was demonstrated by tests performed at respective clinical centers. In addition, in all DNA samples, the $100 \%$ chimerism was confirmed by analyzing genotypes of 27 hypervariable STRs using Fusion 6C kit (Promega).

\section{DNA methylation analysis}

DNA methylation analysis was performed as described previously [4]. Briefly, 2 ug of sample DNA was bisulfite converted using the Qiagen 96-well kit (Qiagen, Hilden, Germany). PCR reactions were carried out in a total volume of $25 \mathrm{~mL}$ to amplify 5 loci in a total volume of $25 \mathrm{~mL}$ (6p24.2), C1orf132 (1q32.2), TRIM59 (3q25.33), KLF14 (7q32.3), and FHL2 (2q12.2). Negative PCR controls were included in each PCR amplification. Pyrosequencing was performed using Pyro Gold reagents on a PyroMark vacuum prep workstation and a PyroMark Q24 instrument, following the manufacturer's instructions. The generated pyrograms were automatically analyzed using PyroMark analysis software (Qiagen, Hilden, Germany). Our approach allowed to define the methylation status of $32 \mathrm{CpG}$ (7 CpGs in ELOVL2, $10 \mathrm{CpG}$ in FHL2, 8 CpG in TRIM59, $4 \mathrm{CpG}$ in KLF14, and 3 CpGs in C1orf132). The positions of these CpG are given in Table 1 in [4].

\section{Statistical analysis}

Correlations were assessed by calculating the Pearson correlation coefficients $(r)$. We also show $r^{2}$ values which indicate the fraction of variance of one variable explained by the other variable as well as $p$ values. The differences in the predicted age and in the methylation status in the donor-recipients pairs were analyzed with paired $t$ test. In the comparison of methylation status of individual $\mathrm{CpG}$ between donors and recipients, Bonferroni correction was applied with the correction factor of 32 (i.e., number of all analyzed $\mathrm{CpG}$ ). Multivariate analysis was performed with

Table 1 Characteristics of HSCT recipients and donors

Mean Standard deviation Median Min. Max. Lower quartile Upper quartile

\begin{tabular}{|c|c|c|c|c|c|c|c|}
\hline \multicolumn{8}{|l|}{ Recipients ( $N=16.37 .5 \%$ of females) } \\
\hline Calendar age & 42.0 & 11.5 & 45 & 18 & 59 & 36 & 50 \\
\hline \multicolumn{8}{|l|}{ Donors ( $N=16.50 \%$ of females) } \\
\hline Calendar age & 33.9 & 13.3 & 28 & 20 & 63 & 25 & 40 \\
\hline Period (days) between HSCT and blood sampling in the recipient & 125.9 & 97.5 & 91 & 27 & 360 & 41 & 202 \\
\hline Calendar age difference (recipient-donor) & 8.1 & 16.8 & 11 & -19 & 28 & -9 & 22 \\
\hline
\end{tabular}


multiple linear regression (lymphocyte numbers and the increase in C1orf132 CpG_1 methylation were independent variables; donor's age was the dependent variable). All calculations were performed with Statistica software package (StatSoft, Tulsa, OK, USA).

\section{Results}

\section{Accuracy of age prediction among donors}

Among donors, there was a strong correlation between the calendar age and the predicted age $\left(r=0.96, r^{2}=0.93\right.$, $p=2 \times 10^{-9}$, Fig. 1a). Mean absolute deviation (MAD) was 3 years with min. and max. of 0 and 7 and quartile range from 1.5 to 5 years. The calendar age of 14 out of 16 donors $(87.5 \%)$ was predicted with an absolute error of
5 years or less. The mean difference between calendar and predicted age was 1 year, with min. and max. of -7 and 7 years, and quartile range from -1.5 to 4.5 years.

Among HSCT recipients, the predicted age did not depend on the recipient calendar age but strongly correlated with calendar and predicted age of the donor.

Among recipients post HSCT, there was no correlation between the calendar and the age predicted from the methylation analysis of the blood DNA $\left(r=0.134, r^{2}=\right.$ $0.018, p=0.62$, Fig. 1 b). Of note, analysis of the recipients' blood revealed that there was a strong correlation between the predicted age assessed by the methylation signature of blood DNA with the calendar age of the donor $\left(r=0.94, r^{2}=0.89, p=4 \times 10^{-8}\right.$, Fig. 1c) as well as
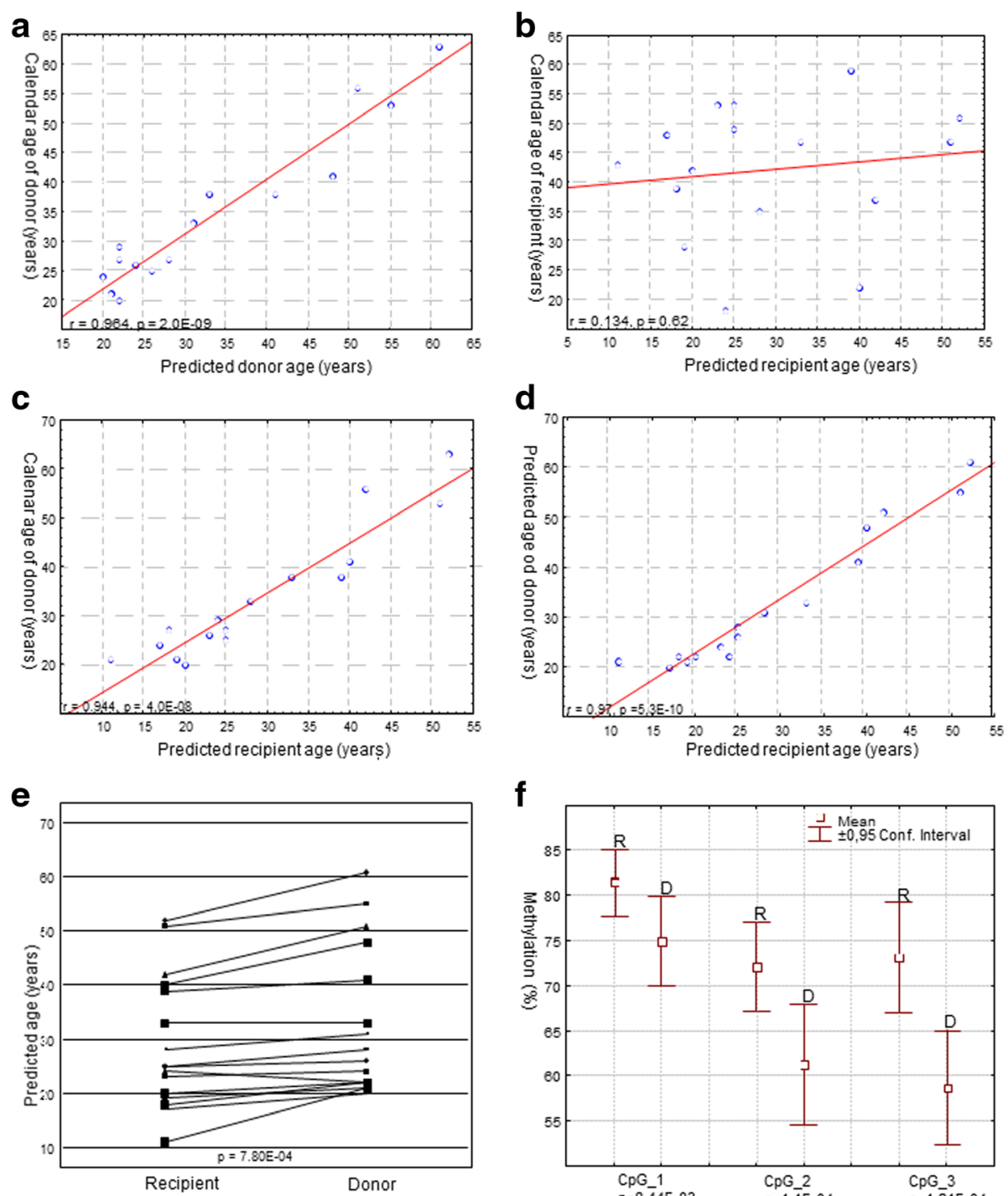

f

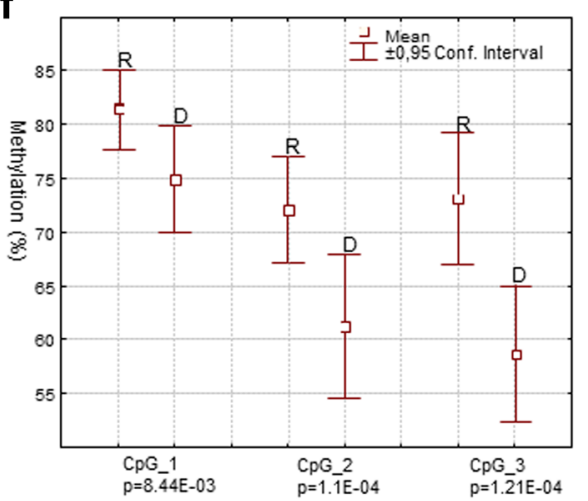

Fig. 1 Correlation between age predicted from methylation and calendar age in HSCT donors (a) and recipients (b). r Pearson correlation coefficient, $p$ associated $p$ value. Correlation between the predicted age of HSCT recipient and calendar age of HSCT donor (c) and predicted age of the donor (d). Age predicted from methylation in the HSCT recipient-donor pairs; $p$ value was calculated by $t$ test for paired samples (e). Methylation levels of the studied C1orf132 CpGs in HSCT recipients (R) and donors (D); $p$ values were calculated with paired $t$ test with Bonferroni correction (f) 
the predicted age of the donor assessed by analysis of the donor's blood $\left(r=0.97, r^{2}=0.94, p=5 \times 10^{-10}\right.$, Fig. 1d).

\section{The methylation level indicates lower predicted age in the recipients compared to the predicted age in donors after HSCT}

Despite the very strong correlation between predicted age of the recipient and the predicted age of the donor, we noted that in all but one case the age predicted from the post-HSCT methylation signature of recipient's blood was lower than the age predicted from the methylation signature in the donor of transplanted material (Fig. 1e). In the case which constituted the exception the age of the recipient predicted from post HSCT DNA methylation status was 2 years higher than the age predicted from DNA methylation status in the respective donor.

The mean predicted age among recipients was 29.2 years with standard deviation (SD) of 12.3; whereas among the donors, the respective value was 32.9 years with SD of 13.81. The mean difference between the predicted age of the donor and the predicted age of the recipient was 3.7 years with SD of $3.52(p=0.00078, t$ test for paired samples).

The apparent lower predicted age in the recipients compared to the predicted age in donors seen at the DNA methylation level (i.e., the difference between the predicted age of donor and the predicted age of recipient) did not depend on sex $(p=0.49)$, the age of recipients $(p=0.95)$, the length of period between HSCT and blood sampling in the recipients group $(p=0.82)$, or the difference in the calendar age between the donors and the recipients $(p=0.14)$. However, we noted a trend $(p=0.064)$ towards a correlation of the size of difference between predicted age in the recipients and donors, with donor's age $(r=0.47)$ suggesting that this effect may be more pronounced when HSCT comes from an older donor (i.e., the older the donor the greater the difference between the his/her predicted age and the predicted age of the recipient).

\section{The apparent lower predicted age in the recipients compared to the predicted age in donors after HSCT is driven by selective hypermethylation of $\mathrm{CpGs}$ within the C1orf132 locus}

We further investigated whether the consistent difference of $\sim 4$ years between the predicted age of recipient and the predicted age of donor was caused by coherent effects of all CpG predictors or the direction of DNA methylation changes was different in particular loci. In Additional file 1: Table S2, we show the results of comparison of recipient vs. donor methylation differences for all the $5 \mathrm{CpG}$ included in the age prediction model as well as for the remaining $27 \mathrm{CpGs}$ whose methylation was assessed in our study. We found that among the CpG sites included in the model, only CpG_1 at C1orf132 (C1orf132 CpG_1) showed different methylation among recipients vs. donors (mean difference $8.2 \%$; $\mathrm{SD}=6.9 \%$ $\left(p=0.00026 ; P_{\text {corrected }}=0.008\right)$.

In order to better estimate the size of this effect, we developed an age prediction model based solely on CpGs in C1orf132. The model included C1orf132 CpG_1 and C1orf132 CpG_3, and it had a reasonable performance $\left(r=0.89, r^{2}=0.8\right.$, and MAD $=6.1$ years $)$. Using the "C1orf132 only" model, the mean predicted age of recipients was 22.4 years and that of donors was 33.8 years, yielding a difference of 11.3 years $(p=0.000016$, paired $t$ test).

Although we did not detect individual recipient-donor DNA methylation differences in other loci than C1orf132, these loci could still have weak effects possibly amounting to detectable joint influence. In order to test this, we developed an age prediction model including all loci except the C1orf132 ( $r=0.96, r^{2}=0.92, \mathrm{MAD}=4.1$ years $)$. Using this "non-C1orf132 model" the mean predicted age of recipients was 32.1 years and that of donors was 33.1 years, yielding a difference of 1.1 year which was not statistically significant $(p=0.28$, paired $t$ test).

\section{Size of the increase in methylation of C1orf132 CpG_1 in the recipient vs. donor correlates with calendar age of the donor}

The size of the increase in methylation of C1orf132 CpG_1 in the recipients vs. the donors correlated with the calendar age of the donors $\left(r=0.76, r^{2}=0.58, p=0.0006\right)$ but not with sex $(r=-0.40, p=0.12)$, the recipient's age $(r=0.13, p=0.62)$, the length of time between HSCT and blood sampling in the recipient group $(r=-0.004$, $p=0.99$ ), or diagnosis (AML vs. other, 9 vs. 6.4 years, respectively, $p>0.5$ ). The size of the increase in methylation of C1orf132 CpG_1 in the recipients vs. the donors nominally correlated also with the recipient-donor calendar age differences $(r=-0.51, p=0.043)$, but this effect was no longer observed after adjustment for calendar age of the donors $(p=0.7)$. Correlation with the age of the donors was also found with the recipient vs. donor age differences calculated using the "C1orf132 only" model $\left(r=-0.7, r^{2}=0.5, p=0.003\right)$.

The increase in the methylation in the recipients vs. the donors was also apparent for two other C1orf132 CpGs studied; for C1orf132 CpG_2, the difference was $13.4 \%\left(\mathrm{SD}=7.54 . p=0.000003, P_{\text {corrected }}=0.0001\right)$ and for C1orf132 CpG_3 it was $13.8 \%$ (SD $=7.8 . p=0.000004$, $P_{\text {corrected }}=0.00012$, Fig. $\left.1 \mathrm{f}\right)$. However, no statistically significant correlation was observed between the size of these effects and the donor's age or other analyzed variables (data not shown). 
The apparent effect of the donor's age on the methylation of C1orf132 CpG_1 in the recipient group could be secondary to the differences in the cellular composition of the reconstituted hematopoietic system in the recipients receiving transplant from young vs. older donors. Indeed, when we analyzed donors age vs. white blood cell (WBC) or counts/percentages of granulocytes/ lymphocytes/monocytes in the recipients, we found that donor's age correlated with lymphocyte numbers in the recipient group $\left(r=0.78, r^{2}=0.61, p=0.0003\right)$. Since lymphocyte numbers in the recipient also correlated with the size of the increased C1orf132 CpG_1 methylation in the recipient vs. donor $\left(r=0.63, r^{2}=0.40, p=0.009\right)$, we analyzed the effect of lymphocyte numbers and the increase in C1orf132 CpG_1 methylation on donor's age by multiple linear regression. We found that lymphocyte numbers and the difference in C1orf132 CpG_1 methylation between the recipient and donor were independent predictors of donor's age (beta $=0.51, p=0.016$ and beta $=0.44, p=0.033$, respectively).

\section{Discussion}

Among human HSCT donors and recipients, we studied methylation in $5 \mathrm{CpGs}$ recently validated by our group for age estimation. We found that the methylation signature of blood post HSCT does not depend on the recipient age but is strongly correlated with the calendar age of the donor. However, despite this correlation we observed that the age of HSCT recipient as predicted from DNA methylation was lower than the age predicted in donors on average by 4 years. Detailed analysis of methylation status of all studied CpG $(N=32)$ showed that this discrepancy was caused by apparently individual effects of CpGs at the Clorf132 locus.

The age prediction among HSCT donors (who can be regarded as healthy subjects representative of general population) independently confirms good performance of our model [4]. The quality of age prediction achieved in the present study (MAD $=3$ years, $87.5 \%$ correct predictions) was comparable to that originally reported ( $\mathrm{MAD}=3.9,71.7 \%$ correct predictions) [4], especially when it was taken into account that majority of the donors were relatively young belonging to age category 20-39 years for which the performance of our model was better (MAD 3.3, $76.7 \%$ correct predictions) than for older age groups [4].

The observation that the age of HSCT recipient predicted by post-transplant DNA methylation analysis performed on DNA isolated from whole blood correlates with donor's but not recipient's age confirms the results of the recent study by Weidner et al. [5]. Both studies are consistent in the conclusion that the hematopoietic niche of the recipient does not noticeably affect ageassociated DNA methylation in blood. It should be emphasized that none of the five CpGs used in our study overlap with any of the three CpG sites analyzed by Weidner et al. [5] which argues that the observed effect is likely to be robust to the exact identity of the set CpG markers used for age prediction.

Despite the very strong correlation between the age predicted for blood in the donor and the recipient, we found that the latter was consistently lower than the former with an exception observed only in a single pair. The difference was relatively small (mean 3.7 years) but it was highly statistically significant. The lower predicted age in the recipients compared to the predicted age in donors by $\sim 4$ years is directly opposite to the observation of Weidner et al. [5] who reported that the epigenetic age predictions were on average 7 years higher than the calendar age of the donor and attributed this finding to stimulation of hematopoietic stem cells in the donor. One difference between the two studies is the time period between HSCT and the recipient's blood sampling: in the study of Weidner et al. [5], it was 1 year whereas in our study it varied from 1 month to 1 year with a mean of 3 months. However, we think it is unlikely that this difference accounts for the observed discrepancy because we did not observe any correlation between the length of time between HSCT and blood sampling in the recipients and the size of the difference between predicted age in the recipients and the donors. Such a correlation should be apparent if the aging of the transplanted hematopoietic cells in the donor was indeed fast enough to shift methylation signature up by 7 years over the time of 12 months as observed by Weidner et al. [5].

Whereas the reasons for the discrepancy between our results and those of Weidner et al. [5] are not clear, it is possible that CpG marker selection is important. When we analyzed CpGs individually we found that the lower predicted age in the recipients compared to the donors after HSCT was exclusively caused by hypermethylation of a single CpG (CpG_1 from the C1orf132 locus) in the recipient vs. donor whereas the remaining four $\mathrm{CpG}$ from other loci used for age prediction did not show changes. Furthermore, the remaining two $\mathrm{CpGs}$ from the C1orf132 locus (which were typed but not used for age prediction) showed even stronger hypermethylation suggesting that the effect was present among all C1orf132 CpGs. This together with the absence of a similar effect among the total of the 29 CpG from the remaining four loci argues against technical artifacts and indicates that genuine differences exist in postHSCT behavior among loci regarding age-dependent methylation. Whereas potentially interesting from a biological perspective, the dissociation of C1orf132 methylation from calendar age in certain circumstances may decrease the practical usefulness of this locus for age prediction. 
Intriguingly, the size of hypermethylation of C1orf132 CpG_1 in the recipients was directly correlated with the calendar age of the donors-the older the donor, the higher the methylation. Since C1orf132 methylation decreases with calendar age, the increased methylation could suggest that at this particular CpG HSCT induces "rejuvenation" which gets stronger with the increasing age of the donor. However, since HSCT from older donors is less successful than from younger donors [6], it is unlikely that what we observe is a genuine rejuvenation with positive clinical consequences. Rather than that we speculate that C1orf132 hypermethylation in recipients of HSCT from older donors reflects some problems with reconstitution of hematopoietic system to the state in which it was present in the donor. This may be linked with delayed or failed restoration of certain types of cells or certain states of their chromatin. It is also possible that senescence-associated methylation changes contribute to the observed effect.

The C1orf132 has recently been shown to encode a long no-coding RNA (lncRNA) which at its 3 ' end includes microRNA MIR29B2C [7]. Whereas the C1orf132 function remains unclear, it is intriguing that in mice, the miR29 family (miR29ab1 and miR29b2c) has been implicated in the self-renewing ability of HSCs as well as determination of organ and body size [8]. Our results suggest that the C1orf132/MIR29B2C locus could also be important for graft function after HSCT and thus should be studied further as a potential graft function/ prognostic marker.

Due to relatively small number of studied subjects our study had limitations. Whereas in our data the correlations between donor's age on one side and C1orf132 methylation and lymphocyte numbers on the other side appeared independent from each other definite conclusions are difficult. Furthermore, we cannot exclude that C1orf132 methylation is a marker of a subset of cells whose development primarily depends on the donor's age and which were not specifically analyzed by us. Studies of these issues are warranted in the future. Finally, our study may not have had sufficient power to detect a significant correlation between the differences in predicted age of donors vs. recipients and the time length from HSCT to blood sample collection.

In conclusion, using an independent set of $\mathrm{CpG}$ markers, we confirm that after HSCT the age of the donor is the major determinant of age-specific methylation signature in recipient's blood whereas the age of recipient does not exert a detectable effect. As a novel finding, we show that in HSCT setting, methylation at C1orf132 has unique dynamics and depends on the age of the donor. The methylation at C1orf132 locus should be interesting for further studies of HSCT prognostic markers.

\section{Conclusions}

After HSCT, the age of the donor is the major determinant of age-specific methylation signature in recipient's blood.

After HSCT methylation of C1orf132, which encodes MIR29B2C implicated in the self-renewing of hematopoietic stem cells, it has a unique dynamics suggesting that this locus could influence graft function.

\section{Additional file}

Additional file 1: Table S1. Calendar age of recipients and matched donors. Table S2. Methylation (\%) of all studied CpGs in recipients and donors. (DOCX $21 \mathrm{~kb}$ )

\section{Acknowledgements}

The authors appreciate the cooperation with the medical team providing all relevant clinical data used in this study.

\section{Funding}

The study is supported by the DOBR/0002/R/ID1/2012/03 grant from the National Centre for Research and Development (NCBiR) in Poland. The work of EJ and AL was supported by a grant INNOMED/I/1/NCBR/2014 from the National Centre for Research and Development (NCBiR), Poland.

\section{Availability of data and materials}

Methylation data and analytical procedures can be provided upon request.

\section{Authors' contributions}

RP and MS conceived the study. MS, AL, TR, WJ, GWB, AW, EJ, and RJ designed the experiments. ŻM, RZP, AP, MJ, AP, JB, WB, and RP performed the experiments and analyzed the data. RP, MS, and AL drafted the manuscript. All authors read and approved the final manuscript.

Competing interests

The authors declare that they have no competing interests.

Consent for publication

Not applicable.

Ethic approval and consent to participate

The study was approved by the bioethics committee at the Medical University of Wroclaw (the opinion of the bioethics committee no. KB-369/2014).

\section{Author details}

${ }^{1}$ Biology Department, Central Forensic Laboratory of the Police, Warsaw 00-583, Poland. ${ }^{2}$ L. Hirszfeld Institute of Immunology and Experimental Therapy, Polish Academy of Sciences, Wroclaw 53-114, Poland. ${ }^{3}$ Lower Silesian Center for Cellular Transplantation with National Bone Marrow Donor Registry, Wroclaw 53-439, Poland. ${ }^{4}$ Department of Hematology, Oncology and Internal Diseases, The Medical University of Warsaw, Warsaw 02-097, Poland. ${ }^{5}$ Department of Forensic Medicine, Medical and Forensic Genetics Laboratory, Medical University of Lodz, Lodz 91-304, Poland. ${ }^{6}$ Department of Hematology, Medical University of Lodz, Copernicus Memorial Hospital, Lodz 93-510, Poland. ${ }^{7}$ Malopolska Centre of Biotechnology, Jagiellonian University, Krakow 30-387, Poland. ${ }^{8}$ Department of Medical Genetics, Warsaw Medical University, Pawińskiego 3c, Warsaw PL 02-106, Poland.

Received: 7 July 2016 Accepted: 24 August 2016

Published online: 06 September 2016

\section{References}

1. Conboy IM, Conboy MJ, Wagers AJ, Girma ER, Weissman IL, Rando TA. Rejuvenation of aged progenitor cells by exposure to a young systemic environment. Nature. 2005;433(7027):760-4.

2. Castellano JM, Kirby ED, Wyss-Coray T. Blood-borne revitalization of the aged brain. JAMA Neurology. 2015;72(10):1191-4. 
3. Bouchard J, Villeda SA. Aging and brain rejuvenation as systemic events. J Neurochem. 2015;132(1):5-19.

4. Zbiec-Piekarska R, Spolnicka M, Kupiec T, Parys-Proszek A, Makowska Z, Paleczka A, Kucharczyk K, Płoski R, Branicki W. Development of a forensically useful age prediction method based on DNA methylation analysis. Forensic Sci Int Genet. 2015;17:173-9.

5. Weidner $\mathrm{Cl}$, Ziegler $\mathrm{P}$, Hahn $\mathrm{M}$, Brummendorf $\mathrm{TH}, \mathrm{Ho}$ AD, Dreger $\mathrm{P}$, Wagner $\mathrm{W}$. Epigenetic aging upon allogeneic transplantation: the hematopoietic niche does not affect age-associated DNA methylation. Leukemia. 2015;29(4):985-8.

6. Kroger N, Zabelina T, de Wreede L, Berger J, Alchalby H, van Biezen A, et al. Allogeneic stem cell transplantation for older advanced MDS patients: improved survival with young unrelated donor in comparison with HLA-identical siblings. Leukemia. 2013;27(3):604-9.

7. Kurinna S, Schafer M, Ostano P, Karouzakis E, Chiorino G, Bloch W, Bachmann A, Gay S, Garrod D, Lefort K, Dotto GP, Beer HD, Werner S. A novel Nrf2-miR-29 desmocollin-2 axis regulates desmosome function in keratinocytes. Nat Commun. 2014:6:5.

8. Kauffman L, Balatti V, Cascione L, Fadda P, Racke F, Santhanam R, Costinean S. Gradual rarefaction of hematopoietic precursors and atrophy in a depleted microRNA 29a, b and c environment. PLoS One. 2015;10(7):e0131981.

Submit your next manuscript to BioMed Central and we will help you at every step:

- We accept pre-submission inquiries

- Our selector tool helps you to find the most relevant journal

- We provide round the clock customer support

- Convenient online submission

- Thorough peer review

- Inclusion in PubMed and all major indexing services

- Maximum visibility for your research

Submit your manuscript at www.biomedcentral.com/submit
Biomed Central 